\title{
LA PUNTUACIÓN EN DOCUMENTOS NOVOHISPANOS. UNA MIRADA DESCRIPTIVA ${ }^{1}$ THE PUNCTUATION IN DOCUMENTS OF NEW SPAIN. A DESCRIPTIVE LOOK
}

\author{
Maribel Delgado García \\ Universidad Nacional Autónoma de México
}

La historia de la puntuación durante el periodo colonial cuenta con pocos estudios. Así, Juan Martínez Marín (1992) apenas alude a ellos, y lo mismo puede decirse de los manuales de paleografía. Ramón Santiago (1998) realizó un trabajo muy completo en el que presentó a los más destacados gramáticos y ortógrafos de los siglos XVI y XVII, y de esta forma señaló el papel de la ortografía y de la puntación en esas centurias.

La Orthographia de López de Velasco (1582) contiene un apartado en el que se habla de la puntuación. Afirma este autor que es de gran importancia saber puntuar un texto, y aunque considera que prevalece puntuar un impreso, asienta que también interesa para la escritura de mano.

En este estudio se presenta una descripción de los signos de puntuación en siete documentos del siglo XVI, con diversa diatopía y distinto registro, basados principalmente en los usos que refiere López de Velasco.

Entre los usos se identifican los de tipo prosódico, sintáctico y semántico: indicar pausa para respirar, separar cláusulas, oponer contenido, introducir explicativas, indicar inicio y fin de periodo. Es posible constatar una mayor variedad de signos en documentos formales, con signos y usos comunes a la Península y a la Nueva España, con preferencia por la función sobre la forma.

Palabras Clave: ortografía colonial, puntuación colonial, puntuación prosódica, signos ortográficos

The history of punctuation during the Hispanic Colonial times has not been sufficiently studied. Juan Ramón Martínez Marín (1992) barely mentions a few works on that subject, as well as about the handbooks of paleography. By contrast, Ramón Santiago (1998) has presented in a thorough work the more remarkable Grammarians and Orthographers of the $16^{\text {th }}$ and $17^{\text {th }}$ Centuries, thus pointing out the important role of the Orthography and Punctuation of that period.

\footnotetext{
${ }^{1}$ Esta investigación se realizó con una beca Conacyt del Posgrado de Maestría y Doctorado en Lingüística Hispánica en el Instituto de Investigaciones Filológicas, y con apoyo del Proyecto Conacyt "Investigación documental, a través de la dialectología y la sociolingüística históricas, para conocer el mestizaje lingüístico en la Nueva España”, CB-2012/180245. Bajo la tutoría de la doctora Beatriz Arias Álvarez.
} 
López de Velasco Orthographia (1582) includes a chapter/paragraph which deals with punctuation. According to him it is of utmost importance to know how to punctuate a handwritten text, although he gives even more importance to the punctuation of a print text.

This paper presents a description of the punctuation signs in seven documents from the $16^{\text {th }}$ Century, considering both the several registers and diatopias in each one of them upon the basis referred by López de Velasco.

Among these types we identify prosodic, syntactic and semantic usages. Some examples are the pause signal for breathe, the order to separate clauses as well as to oppose content, introduce explanatory sentences and point out the beginning or the end of a period. It is possible to note a wider range of signs in formal documents, with common usages for the New Spain and the Iberian Peninsula which stress out the grammar function over the grammar form.

KEYworDs: Colonial orthography, Colonial punctuation, prosodic punctuation, orthographic signs

RECEPCIÓN: 31/10/2016

ACEPTACIÓN: 02/01/2017

\section{Introducción}

Se quejaba Juan Martínez Marín en su artículo "La ortografía española: perspectiva historiográfica”, allá por el año 1992, de la poca atención que había recibido la puntuación dentro de los estudios ortográficos. ${ }^{2}$ Afortunadamente, a la fecha, se cuenta ya con varias investigaciones que han subsanando el estudio de la escritura en los elementos adsegmentales: ${ }^{3}$ M. B. Parkes (1992) y Nina Catach (1994), así como las investigaciones imprescindibles de Fidel Sebastián Mediavilla a partir del $2000 .^{4}$

Desde el comienzo, la (pre)ocupación de los gramáticos por describir y prescribir la escritura del español fue la letra, el grafema en cuanto forma que establece una correlación con un

\footnotetext{
${ }^{2}$ Entre los cuales se encuentra un importantísimo trabajo que llevó a cabo Abraham Esteve Serrano en 1982.

${ }^{3}$ No he encontrado trabajos realizados específicamente sobre los elementos suprasegmentales.

${ }^{4}$ Aunque no se han podido consultar de manera directa los dos primeros, presentamos aquí los datos: M. B. Parkes, Pause and Effect: An Introduction to the History of punctuation in the West, Londres, Scolar, 1992 y Nina Catach, La ponctuation (histoire et systhème), Presses Universitaires de France, 1994.
} 
fonema, en la mayoría de los casos, para el castellano, de manera biunívoca. Parece innegable que ese enfoque letrista de los estudios ortográficos era necesario dada la naturaleza de los segmentos de que se trata $y$, sobre todo, sigue siendo fundamental para todo estudio diacrónico de nuestra lengua, que no puede hacerse si no es a través de los textos.

Margit Frenk anota que, desde que empezaron a surgir las gramáticas del español, la puntuación ocupó un lugar destacado, ${ }^{5}$ ya que algunos ortógrafos estaban convencidos de la pertinencia y las consecuencias que el empleo de esta traía, sobre todo al leer el escrito. Se observa esto no sólo en Mateo Alemán, sino ya desde Cristóbal de Villalón, a mediados del XVI, quien presenta la primera reflexión y recomendación específica de este contenido ortográfico. ${ }^{6}$

La puntuación, desde su origen prosódico y hasta la implicación de aspectos semánticos y sintácticos, se ha ido constituyendo como un complejo sistema que contribuye a la recuperación del contenido textual mediante 'apoyos' para la enunciación y la organización de ideas. Este proceso ha sido observado y expresado por Sebastián Mediavilla (2000: 66) al dar cuenta de cómo los ortógrafos y gramáticos pasaron de una justificación de la puntuación como auxiliar en la lectura - para indicar donde se debería hacer pausa-, a una donde se indicaban enunciados con sentido completo y oraciones gramaticales, o sus partes o miembros, o una combinación de ambos aspectos.

\footnotetext{
${ }^{5}$ Esta finalidad de la puntuación parece estar clara en los gramáticos del Siglo de Oro. Margit Frenk recupera citas curiosas donde se pondera que se escribe para leer. Cita de Juan Pablo Bonet, “de los que leen muy bien latín, pero no lo entienden' (1620: 153) y propone el procedimiento para una fase del aprendizaje de la lectura: 'no ay que reparar en que [el lector] no entienda lo que leyere', porque basta 'que haga letura inteligible para el que lo oyere, aunque no sepa lo que dize"' (1983: 6).

${ }^{6}$ Alejo Venegas, en su Orthographia de 1531, incluye un apartado, la regla XviII, dedicado a la puntuación latina. Expone ahí la finalidad que tiene tintes prosódicos y se considera una especie de glosa: "Digo pues que la punctuacion haze que descanse el que habla: y perciba bien el que oye: y entienda el que lee”. Las señales que declara son seis: Comma (:); colun (.); articulus (:); paréntesis () que en latín correspondía a cuatro puntos (::), los dos primeros eran commata y los segundos la colla; vírgula (/) descrita como acento pero visualmente se trata de una diagonal; y el interrogante.
} 
En los últimos años, como cité líneas arriba, Sebastián Mediavilla (2000) ha dado comienzo a la tarea de estudiar la puntuación en los Siglos de Oro, centrándose en su aplicación y desarrollo en los textos literarios. Este autor analiza el estado en que se entregaba un autógrafo, las modificaciones realizadas en el original de imprenta - que se suponían definitivasy la edición prínceps que, finalmente, imprimía el componedor de obras tales como El Quijote y La Celestina, entre otras.

La interactiva relación entre los estadios del proceso de la imprenta se nos presenta como el punto de partida de nuestro sistema y nuestras normas de puntuación. De lo anterior puede saberse que, si bien la puntuación surge como respuesta a la necesidad prosódica y retórica más propia de la oralidad, ${ }^{7}$ los hechos socioculturales como la vuelta a los clásicos durante el Renacimiento, las ideas filosóficas de humanismo, el desarrollo técnico e industrial emparejado con la imprenta y el consecuente auge en la difusión de los libros, marcaron las pautas para entender y plantear la puntuación desde puntos de vista diferentes al tradicional, aunque sin abandonarlo. ${ }^{8}$

\footnotetext{
7 Véase Rubio Flores (2006); Sebastián Mediavilla (2000); Frenk (1983); Peñalver (2015). Este último autor no ha sido consultado para este trabajo, pero su importancia radica en el detallado y paulatino recuento del Prontuario de ortografía de la lengua castellana.

${ }^{8}$ Y, efectivamente, nos recuerda la última edición de la Ortografía de la RAE que los primeros escritos estaban destinados a la lectura en voz alta, situación que requería especificaciones que proporcionaran a los lectores todo tipo de indicaciones para la lectura correcta de los textos y, en consecuencia, para la comprensión de los escuchas: "Los tratadistas clásicos se refieren en sus obras al mencionado sistema ternario de puntuación; sin embargo, en la práctica, son escasos los textos puntuados y, cuando lo están, los criterios de uso no son uniformes. En ocasiones el sistema de tres pausas pasa a ser binario: este sistema reducido consiste en el empleo de un signo para la pausa fuerte que acompaña a las unidades autónomas de sentido, y otro para la pausa débil separadora de unidades no autónomas desde el punto de vista semántico. Este será el modelo elegido por Elio Antonio de Nebrija, entre otros, en el siglo xv. En la Alta Edad Media se mantiene básicamente el sistema grecolatino de tres signos, aunque en época carolingia se documenta ya el llamado signo interrogativo. Con el incremento del empleo de las letras minúsculas, cada vez se hace más difícil distinguir la altura del punto en el renglón, lo que favorece el surgimiento de nuevos signos. Además, concurren en ese momento ciertos factores que propician el aumento de los textos puntuados, como la labor de los gramáticos (san Isidoro entre
} 
Contextualmente, de una condición social marcada en favor de una élite lectora, se pasó a una sociedad más alfabetizada con un aumento en el número de lectores; de una distribución de libros restringida, a una prácticamente sin límites gracias a la imprenta; de un primer fundamento prosódico-retórico, a otro sintáctico-semántico. Como señala Ramón Santiago, el cambio de un sistema de puntuación con base latina tiene que ver con la hipótesis de un cambio en la concepción de lo que era una cláusula y su división interna. Citamos:

Los cambios específicos en el uso desde el siglo XVIII —más fijo, menos flexible - tendrán que ver, sobre todo, con el modo de entender la adecuación de la división interna de la frase según el sentido a relaciones e implicaciones concretas de orden sintáctico (1998: 271).

\section{También la RAE en su Ortografía nos dice:}

Por otro lado, con la proliferación de ediciones y el incremento de niveles de alfabetización, la lectura pasa de ser una actividad colectiva a realizarse individualmente, de manera silenciosa, lo que da protagonismo al texto como texto escrito e implica cambios en el punto de vista a la hora de puntuar, que progresivamente irá privilegiando los criterios sintáctico-semánticos sobre los prosódicos (2011: 90).

Y si bien con este cambio en la puntuación, que va de la enunciación y la semántica a la estructura gramatical, nos refe-

ellos) o la contribución de personalidades como Carlomagno, gran promotor de la producción y copia de libros de la corte. No obstante, como en la Antigüedad clásica, las funciones de los signos están poco definidas y su uso carece de sistematicidad. Por otra parte, la puntuación continúa ligada a la oralidad y, por tanto, se sigue puntuando principalmente para facilitar la lectura en voz alta; sin embargo, con el desarrollo de las cancillerías y la proliferación de traducciones de la Biblia, una de las funciones de la puntuación pasa a ser también la correcta interpretación de los textos escritos, ya que una mala lectura puede significar un equívoco jurídico, en el caso de los textos cancillerescos, o una herejía, en el de los bíblicos. Lentamente el sistema evoluciona y se van introduciendo nuevos signos, muchos de ellos con una forma parecida al punto y coma, aunque con valores diferentes" (2011: 289-290). 
rimos a un fundamento de la misma que da la apariencia de más fijación o estabilidad, sigue siendo un elemento de la escritura bastante problemático, como bien apunta nuevamente Sebastián Mediavilla:

¿Los signos?: como durante todo el Siglo de Oro, no acaban de tener un valor claramente diferenciado [...] Se trata de guardar un equilibrio entre unos límites infranqueables y unas opciones variadas donde, sin alterar el sentido, cabe, mediante una puntuación optativa, matizar un sentimiento, acentuar una pausa, o poner más claramente de manifiesto las relaciones sintácticas de las partes $^{9}$ (2008: 242).

Pero ¿cuáles serán las características del proceso de puntuar un texto que no está destinado a la publicación, pero que igualmente está destinado a la lectura? Finalmente, como dice Olson: "Un sistema de escritura desemboca en textos escritos que se leen - es decir, son leídos-y desde ese momento se convierten en modelo de lo que se dice" (apud Blanche-Benveniste, 1998: 86).

Si bien lo arriba dicho es el motivo de esta investigación, el objetivo es identificar los recursos puntuantes y describirlos en su aplicación en siete documentos enmarcados en el segundo cuarto del siglo Xvi. Asimismo dar cuenta de si los usos novohispanos se acercan más o menos a determinados usos de la península ibérica - principalmente Sevilla o Madrid-. Pero antes de presentar nuestros documentos y su análisis, consideramos necesario tener presente dos conceptos: el de puntuación, de Juan López de Velasco, autor en quien nos basaremos para comparar normas y usos; y los de cláusula, en Cristóbal de Villalón y en Jiménez Patón; ${ }^{10}$ estos últimos porque refuerzan temporalmente la idea de cláusula en López de Velasco (el primero de 1535 y el segundo de 1614).

\footnotetext{
${ }^{9}$ Así encontramos a Sebastián Mediavilla (2000: 32) recordando: "los seis 'puntos de entonación' suplementarios que reclamaba Hervé Bazin: el punto de ironía, el de duda, el de certeza, el de aclamación, el de amor, y el punto de autoridad".

${ }^{10}$ Estas definiciones siguen vigentes, según se lee en el Diccionario básico de Lingüística, y en el Diccionario de la RAE consultado en línea: www.rae.es.
} 
López de Velasco da cuenta de que con la puntuación los significados son aclarados, se concibe la cláusula como un compuesto de 'partes y miembros' y, además, proporciona información sobre el ritmo de lectura. Nos dice:

saber que la puntuación tiene fin a tres cosas; la primera apartar los miembros de la oración dudosos: la segunda, señalar los lugares, donde el aliento, y voz, hablando, o leyendo, haze pausa: y la tercera, distinguir las cláusulas, partes, y miembros dellas (1582: 287-289).

Para Villalón (apud Sebastián Mediavilla, 2000: 70) la oración tiene carácter sintáctico, mientras que en la cláusula se perciben tanto la semántica, en cuanto sentido completo, como la sintaxis, en cuanto ayuntamiento de muchas oraciones. Cito:

deue notar, que ay differençia entre clausula y oraçion. Que oraçion, a lo menos perfecta, se compone por la mayor parte de persona que haze alguna obra: y de verbo: y de persona en quien se denota passar, o hazer aquella obra del verbo. Como esta oraçion: Yo amo a dios, es perfecta: porque yo soy la persona que haze esta obra de amar, que es la obra del verbo, amo: y Dios es la persona que padece. Y digo, que clausula es a las vezes vna oraçion sola: $y$ otras vezes es vn ayuntamiento de muchas oraçiones: las quales todas juntas espresan y manifiestan cumplidamente el conçibimiento del hombre en el proposito que tiene tomado para hablar.

En cambio, para Jiménez Patón en su Epítome, la cláusula es eminentemente sintáctica, pues aunque 'razón perfecta y acabada', su descripción se enfoca en la división. Escribe:

la cláusula o periodo es una raçón perfeta y acabada [que debe dividirse para que] descanse y haga pausa el que raçona, [se divide comunmente en seis partes]: la primera en inciso, que también se llama coma o medio punto [...] con la qual partimos la cláusula en seis partes más menudas (1614: 75-76).

Finalmente, como dice Ramón Santiago (1998: 271), "los cambios específicos [...] tendrán que ver, sobre todo, con el mo- 
do de entender la adecuación de la división interna de la frase según el sentido a relaciones e implicaciones concretas de orden sintáctico". Es decir, que el origen prosódico pasó a un segundo plano, aunque la RAE (2011) nos dice que en el uso de la puntuación se observa que ambas tendencias, la prosódica o enunciativa, y la sintáctica, coexisten durante el Renacimiento.

a. Puntuación prosódica o retórica proveniente de la tradición grecolatina tenía como fin la lectura en voz alta, por lo que se privilegia el aspecto enunciativo.

b. Puntuación lógico semántica es consecuencia de la lectura en voz baja, dando prioridad a las unidades sintáctico-semánticas.

Sebastián Mediavilla (2007: 29) nos muestra en las conclusiones de su estudio sobre la puntuación en el Quijote los distintos usos de que es objeto la puntuación. Enumera los siguientes tipos de normas, según se relacionen con:
c. la estructura de la frase
d. la prosodia
e. determinadas palabras (entre ellas las conjunciones)
f. determinadas funciones
g. la tipografía

Por otra parte, un buen trabajo donde se pueden ver las diferentes propuestas de signos de puntuación y usos es el de Ramón Santiago (1998). En este artículo se toma nota de la introducción de la coma, el punto y coma y los dos puntos por Aldo Manuzio El Viejo, impresor italiano; y El Joven agrega el apóstrofo y el acento. Antonio de Torquemada emplea, además, el guion y la diagonal.

Algunos de estos signos tienen dos funciones, por ejemplo, para Venegas los dos puntos se denominan 'artículo' cuando se ponen en lugar de conjunción, y mantienen su nombre de 'dos puntos' si dividen la cláusula en dos; también emplea la diagonal combinada con punto cuando "sirve de artículo [cuando] la sentencia es muy imperfecta porque no hay verbo y es necesario to- 
mar un huelgo insensible que no sea tan behemente como el de la comma”. Madariaga y Nunes de Lião llaman 'vírgula' a lo que denominamos 'coma', y Nunes dice que emplean los dos puntos cuando refieren palabras de otro. ${ }^{11}$

Por su parte, López de Velasco (1582: 286) reconoce el apóstrofe, el acento circunflejo y el agudo, los signos de admiración e interrogación, el espacio en blanco entre palabras, el ayuntamiento mediante el iphen $(\sim)$, y el uso de mayúsculas, además de:

- $\quad$ El punto redondo, periodo o final (.)

- La diagonal que él llama rasguillo, vírgula y rayuela larga (/)

- Dos puntos o colon (:)

- La coma (,) que denomina como medio punto, incisión, cortadura menuda

- Del punto y coma (;) no proporciona nombre

- Del paréntesis ()

Distingue entre los signos para usar en impreso y los de manuscrita porque "la mucha differencia de puntos parece que desmiembra la oración, o a lo menos que embaraça el hilo, y corriente della: y también porque no carece de difficultad, saber dividir las cláusulas y miembros dellas, conforme a razón”.

Los signos que permanecen para la escritura de mano son:

- Coma o medio punto (,) para distinguir "las partes pequeñas: donde si se cortasse la oración, quedaría imperfecta, y suspensa”.

- Colon o dos puntos (:), para distinguir "los miembros más principales, donde se quisiere dar a entender que la razón passa adelante".

\footnotetext{
${ }^{11}$ Otros autores que se registran son Nebrija con su sistema binario; Francisco de Robles que habla de articulus o comma, pero también de punto cuando hay numeración. En 1543, para las obras de Garcilaso impresas en Barcelona, se usa (.), (,), (:). Para Pastrana, la diagonal funciona como puntos suspensivos. Con Juan de Ycíar cambia el sistema anterior al incluir la coma, los dos puntos y el punto. En la impresión de Cristóbal de Villalón emplean también el punto y coma.
} 
- Punto redondo, final o periodo (.) donde "la razón, o cláusula se cerrare".

Pero incluso pueden reducirse a solo dos:

- Punto entero y rayuela atravesada (./) para "fin de las cláusulas y razones".

- Coma o medio punto (,) en "las otras partes donde se offreciere duda, o se hubiere de alentar".

De lo anterior es importante resaltar dos puntos: el primero, que Velasco puntualiza la dificultad que les representaba la división correcta de las cláusulas; lo segundo, la consideración específica de que para la manuscrita no es necesario más de unos cuantos signos, muchos menos que para los impresos.

\section{Propósito de la investigación y análisis}

Como hemos podido observar a lo largo de las páginas anteriores, la puntuación no es un tema innecesario para el estudio de nuestra lengua española; aunque los primeros ortógrafos le prestaron poca atención, es necesario, para tener un conocimiento más completo y fidedigno del español y de su evolución, que se ponga atención en este aspecto.

López de Velasco dedicó una amplia segunda parte de su Ortographia a este tema, y de alguna manera adelantó los tres principios de la ortografía que conocemos ahora; pero, además, planteó un sistema de puntuación fundamentado en la prosodia, la semántica y la sintaxis, que da cuenta de los usos de sus coetáneos. Dicho autor promueve el empleo de un conjunto de signos para la escritura de mano, que llega a reducir a dos. Pero ¿cuáles de estos signos son empleados en la documentación de los Siglos de Oro y con qué frecuencia?

Hemos analizado siete documentos del segundo cuarto de siglo XVI, cuatro de ellos americanos y tres peninsulares, todos ellos localizables en el corpus electrónico del español COREECOM. El contraste que se busca observar es diatópico — no solo las 
diferencias continentales, sino las variaciones entre áreas influyentes como la corte madrileña, por un lado, y Sevilla, por otro- y diafásico, es decir, las variantes que se dan según el grado de distancia comunicativa (Koch y Oesterreicher, 2007) que presenta el manuscrito.

\begin{tabular}{|c|c|c|c|}
\hline Doc. & Año & Diatópico & Registro \\
\hline Nuño de Guzmán ${ }^{12}$ & 1537 & Nueva España, México & Semiformal \\
\hline Lic. Sandoval & 1539 & Nueva España, México & Semiformal \\
\hline $\begin{array}{l}\text { Obispo de Guate- } \\
\text { mala }^{13}\end{array}$ & 1541 & $\begin{array}{l}\text { Nueva España, Capitanía Gral. de } \\
\text { Guatemala }\end{array}$ & Formal \\
\hline $\begin{array}{l}\text { Obispo de Guate- } \\
\text { mala }\end{array}$ & 1548 & $\begin{array}{l}\text { Nueva España, Capitanía Gral. de } \\
\text { Guatemala }\end{array}$ & Formal \\
\hline Leonor del Río & 1526 & Península, Sevilla & Informal \\
\hline Juana Vázquez & 1538 & Península, Trujillo & Informal \\
\hline Reyna $^{14}$ & 1536 & Península, Madrid & Formal \\
\hline
\end{tabular}

Los signos o señales que pueden interpretarse como marcas de puntuación en los escritos arriba mencionados son: el punto (.), la diagonal sola o con punto (/), (./), (./.), (.//.), los dos puntos (:), paréntesis ( ), guion bajo ( $\_$) y el espacio en blanco. Lo que presentamos a continuación son los usos de cada uno de estos puntualizadores documentados.

\section{Punto}

Aunque no se considera signo de puntuación cuando indica abreviación, queremos señalar que es el empleo más regular que tiene esta señal (ej. 1). Como signo de puntuación acota oraciones explicativas o miembros de la oración (ej. 2), uso que está

\footnotetext{
${ }^{12}$ Nacido en Guadalajara, España, municipio de Castilla.

${ }^{13}$ Se trata del obispo Francisco Marroquín que nació en Santander, España.

${ }^{14}$ La reina Juana I, llamada Juana La Loca.
} 
bien señalado por López de Velasco e identificado por Sebastián Mediavilla; se emplea en enumeraciones (ej. 3); separa enunciados con sentido completo (ej. 4); puede aparecer al final de un párrafo (ej. 5), aunque solo se observa una vez; de manera enfática separando una causal (ej. 6.a); en otra ocasión (ej. 6.b), aisla el sintagma 'sin hablar' - posiblemente subrayando la entonación- y en 6.c pausa un pronombre relativo. Es de notar que este signo no se emplea en el documento de Leonor del Río, de Sevilla.

Como marca de abreviación se observa en todos los documentos: en Nuño de Guzmán, en el Obispo de Guatemala y en Juana Vázquez, de Trujillo.

Ej. 1 1. ILLmo.Rmo.Sor 2. muy magcos.Sres.

- $\quad$ 1. S. C. C. mt.

- 2. /por ebitar confusion que Conla variedad desu p'ceder suelen Cau sar

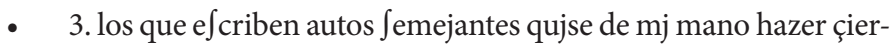
to a.V. mt

- 21. Seruida la yglesia Baotan Al presente Dean Arcediano thesorero Que

- 22. Eøtan aCa / y si v'ra. mgt. fuere seruido de mequerer hazer md. de

- 2. Joana vazquez parezco ante .v. mçd y digo q' puede av'

El licenciado Sandoval, Nuño de Guzmán y la Reina acotan oraciones explicativas (oe) o un miembro de la oración.

Ej. 2 oe

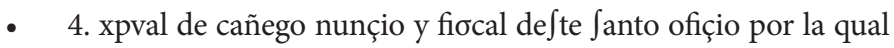
me acu a aver yo judayçado djcho

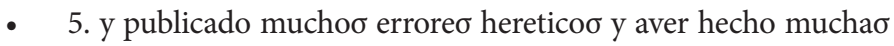
çerjmonja $\sigma$ judaycas en ofensa de n'ra

- 6. Janta fe catolyca. Jegund mao larga mente en la djcha acu $\int \mathrm{\int}$ yon $\int \mathrm{e}$ contiene. el thenor dela

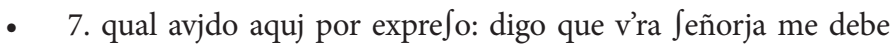
mandar dar por libre y qujto delo 


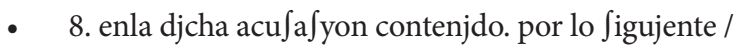

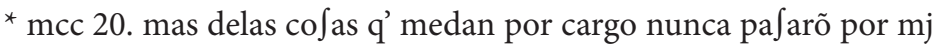

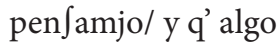

- 21. paso. por la coftumbre. que ellos teniã de dallo de $\int u$ voluntad/ y no hecho

- 4. atenta laqualidad desupsona Alo quenos Auia seruido enla ysla decuba ẽla

- 5. rresidençia quefue Atomar algouernador yoffiçialeo della. E lo que enello Auia

- 6. gastado acuya causa venia muy pobre. le encomendo çiertoo yndios / los qualeo.

Es empleado en una lista (ls) por el Obispo.

Ej. 3 ls coord

- 24. /la Casa de franco lopez rregidor derribo la mayor parte y enella mato

- 25. treyntay seys personas entre me $\int$ tizos. negros E yndios. efCaparon

- 26. vivos ely su muger.y eftando ambos Jumidos enel çieno aftalos

- 27. Sobacos.dize<tachado $>$ que llego aelvn. negro elmas alto quele peresçe av'

Separar oraciones y cláusulas cuando el sentido está completo. Esto lo hacen tanto Nuño como el Obispo.

Ej. 4 cla

- 35. + jufto sera que noo acordemoo de hazer Algun seruicio a .v'ra mgt.

- 36. Temporal y dar auiso cerca dello y a $\iint i$ digo Que Si .v. mgt. [6] 1. Quiere Algun prouecho de fta tierra y mucho Como Es Razon y Seleden

- 2. Mande v'ra mgt. meter En eftas prouicias cinco o seis mjl negros Que Se

- 3. an Buenos Podrase hazer la Contratacion A poca cofta Con Alfonso 
- 4. de Torreo. En eota tierra ay mucho oro y plata Para EJto Eo me

- 5. nefter Que .v. mgt. Mande Que dela eopañola Saquen treo oquatromjl

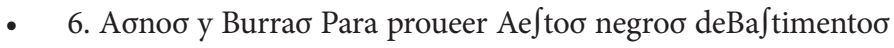
y a

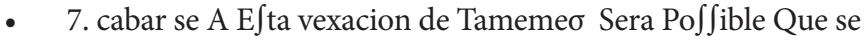

- 8. Ria Efte mj pareocer Pero yo prometo a .v. mgt. que para su Real

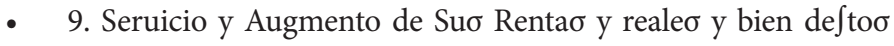
Natu

- 10. raleo que no ay Cofa que se pueda proueer tan Buena y tan

- 11. acertada y Sinque .v. mgt. ponganada de su Casa Que todo se

- 12. Conuierte En su Real Jeruicio

Aparece a final de párrafo, aunque este empleo también se observa una sola vez en el escrito del Obispo.

Ej. $5 \mathrm{cl} /$

- 9. + Si se oviese de hazer Loquequieren Loo eøpañoleo o desean Seguirseha vn yn

- 10. Coueniente muy grande En deo? seruiciodedioo n'ro Señor y del deo cargo

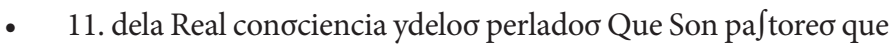
Como Loo Cura $\sigma$

- 12 fueSen pueotoo por Su mano y a $\int \mathrm{u}$ voluntad no podriamoo Loo perladoo

- 13. Ser acusado $\sigma$ de Como Son tractadoo Loo naturaleo de su Encomendero

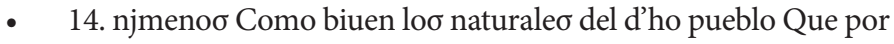
no ser ex

- 15. pellido oyra y vera y Callara prouea .v. mgt. Loque mao fuere Seruido.

Aparece en un par de ocasiones con uso enfático; este uso se encuentra en Nuño de Guzmán (es de anotar que su carta busca conmover) en un caso que es actualmente obligatorio, después de conjunción (marcador discursivo) (ej. 6.a); o aislando sintagmas (ej. 6.b) - lo que hace el Obispo- o enfatizando, como hace la Reina. 
Ej. 6.a enf. después de conjunción

- 5. te. en tanta aufençia de mj Rey y Sor. mandandome ẽzerar entre eftas pare

- 6. des con los mal hechores/ prohibiendome todo adjutorio y recurfo humano

- 7. q' deuia tener/ aunq' hereje y traydor fuera / pues. teniendo quiẽ reprefen

- $\quad$ 8. te la pa. de Ju mt y haga Jufta. ene $\int t a s$ ptes./ ha de remitir lo q' ami tocay $\mathrm{e}$

Ej. 6.b aisla sintagma

- 26. vivos ely su muger.y eftando ambos fumidos enel çieno a)talos

- 27. Sobacos.dize<tachado $>$ que llego aelvn. negro elmas alto quele peresçe av'

- 28. vifto en su vida y que sin. El hablar. le pregunto syhera morales

- 29. arriba d'ho y lerrespondio que no y Como thenja vna viga dela Casa

- 30. atrabesada envn braço que thenja fuera. lerrogo sela qujtase lo

Ej. 6.c pausa pronombre relativo

- 6. gastado acuya causa venia muy pobre. le encomendo çiertoo yndios / $\underline{\text { los qualeo. }}$

- 7. nuño deguzman Elos liçençiados matienço ydelgadillo lequitarõ sincau $\int a$ alguna

\section{Diagonal}

Puede emplearse sola (/) o combinada con puntos (./), (./.), (.//.), (/.), como indicó López de Velasco. Podemos encontrarla en los siguientes casos: separando oraciones o miembros (ej. 7); puede indicar comienzo y fin de párrafo (ej. 8) —es de advertir que en posición inicial alterna con una cruz y en posición final con un guion bajo-; aparece en fin de cláusula (ej. 9) cuando un sentido está completo y debe seguirse con otra cláusula de manera inmediata; antecede a las completivas (ej. 10); puede tener 
un uso enfático (ej. 11); también separar prefijos o palabras en fin de línea (ej. 12) — aunque estos son infrecuentes-; y seguir al saludo (ej. 13). En el documento de Juana Vázquez no se usa este signo.

Son Nuño de Guzmán, el Obispo y la Reina quienes separan oraciones.

Ej. 7 separa oraciones

- 5. te. en tanta aufençia de mj Rey y Sor. mandandome ẽzerar entre e $\int$ tas pare

- 6. des con los mal hechores/ prohibiendome todo adjutorio y recurfo humano

- 7. q' deuia tener/ aunq' hereje y traydor fuera / pues. teniendo quiẽ repre $\int e n$

- $\quad$ 8. te la pa. de $\int u$ mt y haga Jufta. ene tas ptes./ ha de remitir lo q' ami toca y $\int e$

- 9. pide en mj defensa/ forzadome/ es lo que de palabra explicar no puedo

- 10. dezir algo dello por carta/ Juplicando adios n. Sor. como a) oberano Juez q' co

* separa oraciones

- 27. / Cayeron otros muchos pedaços de Casas yentrellos vna por tada y Cuarto dela

- 28. mja la qual mato avnbachiller Contreras / y delas demas la mayor parte eftan

- 29. tan açolbadas de çieno que no se pueden abitar. final men te murie

* separa oraciones

- 2. + Andando visitando Eotas prouincias Jupe Comoauia desembarcado Elldo cerrato

- 3. nuevo presidente / y Como me hallecerca vine Auisitalle / pareoceme que traebue

- 4. noo deseoo deCumplir loo mandamjentoo de v'ra. mgt. y dehazer juftiçia / y

- 5. a tan buen Zelo deCreer Eo que dios n'ro señor le ayudara y fauoreocera

- 6. gastado acuya causa venia muy pobre. le encomendo çierto $\sigma$ yndios / los qualeo. 
- 7. nuño deguzman Elos liçençiados matienço ydelgadillo lequitarõ sincau $\int$ a alguna

- 8. eotando auoente elmarqueo del valle Eporque leayudaua ensuopleitos comosu /

- 9. letrado lehizieron muchas vexaçioneo prendiendole y desterandole yamenazandole

- 10. como todo ellonos Auia conftado por çiertos testimonioo Eproçeßos q’anteloo del

En los documentos de Leonor del Río, del Obispo y de la Reina los encontramos indicando inicio y/o fin de párrafo. El último caso es el de la alternancia con la cruz y el guion bajo.

Ej. 8

- $\quad$ 9. / lo prjmero por que el djcho xpval de cañego nunçio fiocal no fue nj e $\sigma$ parte para ponerme la djcha

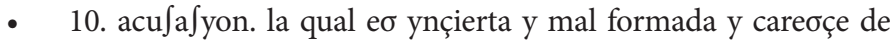
rrelaçion verdadera. njegola en todo

- $\quad$ 11. y por todo como enella $\int$ e contiene afjrmandome en todo lo que tengo djcho y confe $\int a d o$ en la

- $\quad$ 12. confe Jyon que por v'ra señorja me fue tomada /

* 7. /la Casa dexpoual saluatierra all's? yenella no murieron mas de ocho yn

- $\quad$ 8. dios de serujçio y vna donzellica .//.

29. + y tem dela Ropa Que Es Cosa Enq' loo eopañoleo no ponen cofta

- $\quad 30$. nj trabajo jufto sera que paguen decima dela mjoma Ropa Como

- $\quad 31$ y dela Manera que la Reociben njngun agrauio Se leo haze y

- 32. pagarlo En algodón Eo nada /.

* 1 A florentina del

- 13. rrio/.

2. + Andando visitando Eotas prouincias $\int$ upe Comoauia desembarcado Elldo cerrato

- 3. nuevo presidente / y Como me hallecerca vine Auisitalle / pareoceme que traebue

- 4. noo deseoo deCumplir loo mandamjentoo de v'ra. mgt. y dehazer juftiçia / y 
- 5. a tan buen Zelo deCreer Eo que dios n'ro señor le ayudara y fauoreocera

- 6. y Aloque he Conoocido En eotoo pocoo diao Seme representa que e $\sigma$ vna Buena

- 7. maßa De hombre y de Quien Se puede fiar Mucho Caudal

- 17. fecha enmadrid A xxviijo dia $\sigma$ delmeode henero demill Equinios $\mathrm{E}$

- 18. treinta E seys añoo /

Leonor del Río y el Obispo pueden indicar fin de oración o cláusula e ir seguida inmediatamente de otra.

Ej. 9

- 1. + Los officialeo de v'ra mgt. Se An pue to Endezir que elloo son patroneo y que

- 2. ande nombrar Cura $\sigma$ para Lo $\sigma$ puebloo q' eftan En Cabeça de .v. mgt. y aũ

- 3. El audiencia no A querido aclararse y loo vezinoo que tienen yndioo en

- 4. comendadoo dizen lo mjomo que Ande tomar Curao Asu voluntad y qua?

- 5. si qa? la mja .v. mgt. Sea Seruido de ymbiarleo A mandar loque An de hazer

- 6. para q' lo entiendan / e too Son Curadoo propiamente y pueo v'ra. mgt. no se quje

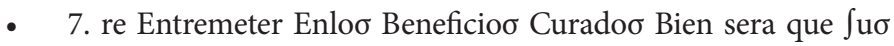
officiale $\mathrm{y}$ loo

- 8. dema Encomenderoo Lo temen y tengan por Cofa Suya

* 12. q'ndo $\int \mathrm{u}$ tio el ob'po de cuenca e $\int$ tatua en roma me enbio $\int \mathrm{u}$ $\mathrm{mt}$ atomar çier

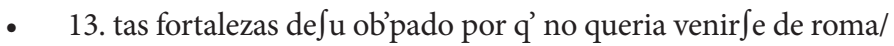
y por q' elp’uior?

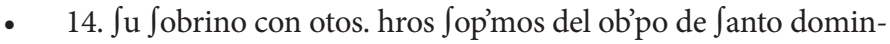
go no quifierõ obedezer

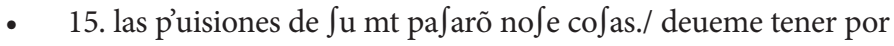
efto algund ran 


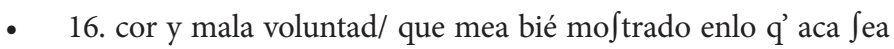
ofrezido y fienp'

- 17. deue auer ef crito o jnformado lo q'le aura parezido p'a q' venga ae tos meri

- 18. tos./ y viendo agora venir vn hombre por Juez dela t'rra del marques./ y egund

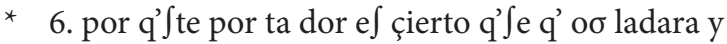

- 7. oo A de buocar / como mj ermanaCatalina del rrio / ef

- 8. falle çida Av'ra? bien çincoAños ./. y v'ro marido

- 9. av'ra? vn Año / ya / yo oo ef crevi otra veo / q'hera

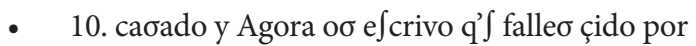

En la escritura del Obispo, Leonor del Río y la Reina se aprecia la separación de oración completiva.

Ej. 10

- 33. no siento otro mejor Remedio nj pienso que lo ay tal para q' v'ra mgt. pueda

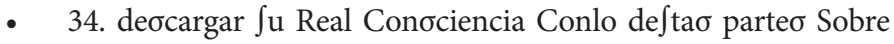
mj Anima que si

- $\quad 35$. .v. mgt. Lo manda proueer Que en t'poo venideroo Se Conozca Loq' agora digo mj

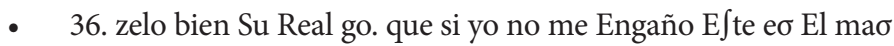
alto E mejor medio

- 37. para deocargo delo paSado preSente y por venir / Con efto descargo pue $\sigma$ ma $\sigma$

- 38. no puedo -

* explic completiva

- 5. Anbas ẽla $\sigma$ caбa $\sigma$ deleonor gtte $\sigma$ v'ra Comadre la $\mathrm{d}<\mathrm{e}>$

- 6. Ayala se os ẽcomjenda / mj matido se os ẽcomjenda

- 7. por Agora no ma $\sigma a l v o$ ? q'damoo rrogan do efto <a>

- 8. dios q'medexeveros / como yodeseo / fecha A xxvi

- 9. dehenero demvxxvj Años /

- 15. vos mando queveais lo sufo d'ho Ellamadao Eoydao laopartes A quien toca hagais

- 16. sobrello breue mente juftiçia /. por manera. q’ninguna dellao rreçiba Agrauio / 
- 17. fecha enmadrid A xxviijo dia $\sigma$ delmeode henero demill Equinios $\mathrm{E}$

- Este es el signo que elige Leonor del Río para dar énfasis en algunas partes de su carta, 'como cuando queda el ánimo suspendido'.

Ej. 11 vocativo?

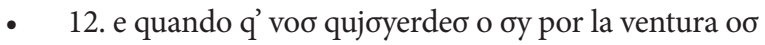

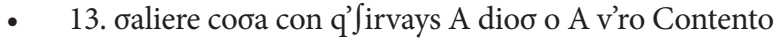

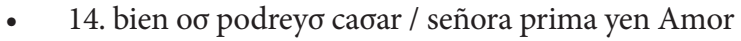

- 15. hera pue q'ya dios me llevola mja bien oabeys

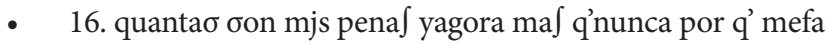

- 17. llo oola ydeo/Abrigada y muj ma oubjeta q’nun

- $\quad$ 18. ca por que bien oabeyo qujen ef mj matido ylo q’yo

- 19. conel pafava en vida demj hermana e v'ro t’po y loo

- 20. Años / meAn oubçedido tales /. q’nj Ai camjøa q'l cuerpo /

- 21. me q'de y mj hermana lohizo cõmigo q'l dioo gelo per/

- 22. done por q’todo loq'tenja lo dexo A Alo de vera y

2. muncha $\sigma$ cat' os he e $\int$ crito y de njnguna e Avido

- 3. rreo.pue ta yonose Aqujen culpe nj se oyculpe A

- 4. qujen / noo la da o A voo por nonenbraros de mj

- 5. como yome Anjenbro devos y Agoravos fizo oaber

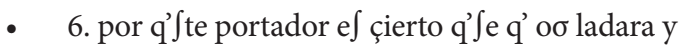

\section{También es Leonor del Río quien lo usa para separar prefi- jos y palabras en fin de línea.}

\section{Ej. 12 Separa prefijo}

- 14. bien oo podreyo caбar / señora prima yen Amor

- 15. hera pue q'ya dios mellevola mja bien oabeys

- 16. quantao oon mjs penal yagora mal q'nunca por q' mefa

- 17. llo oola y deo /Abrigada y muj ma oubjeta q'nun

- 18. ca por que bien oabeyo qujen ef mj matido ylo q’yo

- 19. conel pafava en vida demj hermana ev'ro t’po yloo

- 20. Años / meAn oubçedido tales /. q'nj Ai camjoa q’l cuerpo / separar palabra en fin de línea

- 21. me q'de y mj hermana lohizo cõmigo q'l dioo gelo per/

- $\quad$ 22. done por q'todo loq'tenja lodexo A Alo deveray

- 23. lo hizo heredero yamj nomedexo oyno muncha do 
Asimismo se emplea en el encabezamiento de una carta.

Ej. 13

- 1. deseada señora prima/.

- 2. munchao cat' os he efcrito y de njnguna e Avido

- 3. rreo.puefta yonose Aqujen culpe nj se oyculpe A

\section{Dos puntos}

Son comunes en el licenciado Sandoval y su función es introducir discurso indirecto, aunque se colocan después del verbo de lengua.

Ej. 14

- 5. y publicado muchoo erroreo hereticoo y aver hecho muchao çerjmonja judaycas en ofensa de n'ra

- 6. Janta fe catolyca. Jegund mao larga mente en la djcha acu $\int \mathrm{a}$ yon $\int \mathrm{e}$ contiene. el thenor dela

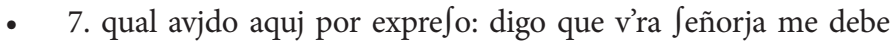
mandar dar por libre y qujto delo

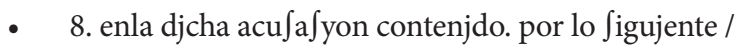

\section{Paréntesis}

Aparece solo en el documento del Obispo de Guatemala fechado en 1548 para acotar oraciones explicativas.

Ej. 15

- 22. Enque muchoo e $\int$ pañoleo tienen por officio de Reocatar gran

- $\quad$ 23. Cantidad DeCaCao q’ É El mayor y mao principal diezmo de $\sigma$

- 24. te ob’pado yComoloo yndioo no pagan decima (yno seria grande

- 25. ynconuenjente pagarlo) noloquieren Elloo pagar y pueo El d'ho 
- 26. CaCao deue El diezmo y viene a poder deloo eopañoleo Que sa

* 32. Entiende En deocargar $\int u$ Real Conociencia y si oviera tenido poo

- 33. sibilidad (como fuera razon que la tuviera) Mao abundantemẽte

- 34. la ouiera deocargado /.

\section{Guion}

Indica fin de párrafo (ej. 16) en el documento del Obispo de Guatemala (1548) y división de palabra en fin de renglón (ej. 17). Aunque debemos decir que se encuentra también antes de conjunción (ej. 18).

Ej. 16 fin de párr

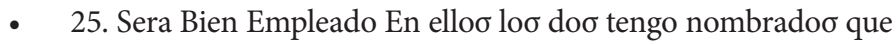
Son El ba

- 26. chiller Joan de Rojao y El Bachiller Joan Çuarez Buenoo grammatico $\sigma$

- [3] 1. Elatinoo E interpreteo para Eotoo Naturaleo que eo delo Que mao neoce $\iint i d a d$

- 2. Tenemos En todo se Cumpla La Voluntad De v'ra. Mgt.

Ej. 17 divide palabra

- 20. de hazer efte Repartimjento Que preserue El mejor pueblo para poder ym

- 21. biar aCa vn buen gramatico vn Buen Artifta vn Buen Theologo

- 22. y vn Buen Canonifta Que facilmente Sepodran sacar dela $\sigma$ vniver

- 23. sidadeo de alcala y Salamanca y que Se asiente vn studio Amane

- 24. ra de vniuersidad En la ciudad de Santiago de guatimala que E $\sigma \mathrm{ma} \sigma$, 
Ej. 18 antes de conj

- 14. Nombre del señor tengo paramj Que En la jo jndía no ay prouja.

- 15. Tan Asentada En la $\sigma \mathrm{Co} \int a \sigma$ dela fe deDioo N'ro señor _ y lealtad

- 16. De su principe y Enel Amor y charidad de suo proximoo Como sõ

- 17. lao prouincias de guatimala. Nunca pretendi mj yntereo Jino El dedioo

\section{Espacio en blanco}

Quien proporciona significado a los espacios en blanco es el obispo de Guatemala. Esto se observa en los dos documentos: puede indicar fin de cláusula o anteceder a una completiva (ej. 19); aislar un pronombre relativo (ej. 20); o anteceder a una subordinada (ej. 21).

Indicando fin de oración o cláusula seguida inmediatamente de otra.

Ej. 19

- 25. ynconuenjente pagarlo) noloquieren Elloo pagar y pueo El d'ho

- 26. CaCao deue El diezmo y viene a poder deloo eopañoleo Que $\mathrm{sa}$

- 27. ben que Lo deuen pueo aquella Cofa noefta dezmada pareoce que

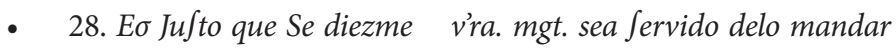

Ej. 20

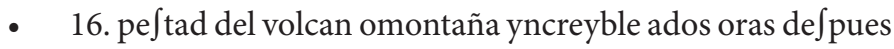
de anocheçi'

- 17. do sabado que hizo eldaño que pensar detraer ala memoria p’a efcrebir

- 18. me El Causa de nueva turbaçion Elqual efplicare por los vrebes termj 
- 19. nos que pudiere y por q' dezirlo que avino avnos y como murieron yse

- 20. hallaron yde Cada vno en particular seria darle titulo de p’çeso y porq'

Ej. 21

- 7 . En que eftaba quenodeujera por que solo Efte rretraimjento quedo en pie

- 8. detodalaCasa y se fue al oratorio que thenja En otra Camara con

- 9. la mayor parte delas donzellas que conella sehallaron que heran doña

- 10. ema mexia y franca desan m’jn Emaria de molina todas tres deubeda

\section{Ningún signo interior}

Es también en los documentos del obispo de Guatemala donde hay párrafos enteros sin más puntuación interna que los puntos de las abreviaturas.

- 9. + Si se oviese de hazer Loquequieren Loo eopañoleo o desean $\int$ eguirseha vn yn

- 10. Coueniente muy grande En deo? seruiciodedioo n'ro Señor y del de $\sigma$ cargo

- 11. dela Real conociencia ydeloo perladoo Que Son paftoreo que Como Loo Cura $\sigma$

- 12. fueSen pueotoo por Su mano y a $\int \mathrm{u}$ voluntad no podriamo $\sigma$ Lo $\sigma$ perlado $\sigma$

- 13. Ser acusadoo de Como Son tractadoo Loo naturaleo de su Encomendero

- 14. njmenoo Como biuen loo naturaleo del d'ho pueblo Que por no ser ex

- $\quad$ 15. pellido oyra y vera y Callara prouea .v. mgt. Loque mao fuere $\int$ eruido.

En los ejemplos vistos, reconocemos las funciones ya indi- 
cadas anteriormente: prosódicas, semánticas, sintácticas y tipográficas; y podemos ver con qué signos fueron señaladas; pero es preciso subrayar que no hay una separación tajante entre las funciones.

Tenemos, por ejemplo, que los signos que pueden indicar un aspecto semántico (hablando de sentidos) son: punto, diagonal y espacio en blanco.

\section{Ej. 20}

- 25. ynconuenjente pagarlo) noloquieren Elloo pagar y pueo El d'ho

- 26. CaCao deue El diezmo y viene a poder deloo eopañoleo Que sa

- 27. ben que Lo deuen pueo aquella Cofa noefta dezmada pareoce que

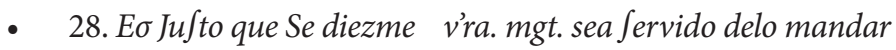

Para la función prosódica se emplea el espacio en blanco, el punto y la diagonal (sola o combinada).

Ej. 6.b aisla sintagma

- 26. vivos ely su muger.y eftando ambos Jumidos enel çieno a)talos

- 27. Sobacos.dize $<$ tachado $>$ que llego aelvn. negro elmas alto quele peresçe av'

- 28. vifto en su vida y que sin. El hablar. le pregunto syhera morales

- 29. arriba d'ho y lerrespondio que no y Como thenja vna viga dela Casa

- 30. atrabesada envn braço que thenja fuera. lerrogo sela qujtase lo

Ej. 21

- 16. peftad del volcan omontaña yncreyble ados oras defpues de anocheçi'

- 17. do sabado que hizo eldaño que pensar detraer ala memoria p’a e crebir 
- 18. me Ef Causa de nueva turbaçion Elqual efplicare por los vrebes termj

- 19. nos que pudiere y por q' dezirlo que avino avnos y como murieron yse

- 20. hallaron yde Cada vno en particular seria darle titulo de p'çeso y porq'

La sintaxis se registra mediante punto, diagonal, paréntesis y espacio en blanco.

Ej. 7 separa oraciones

- 27. / Cayeron otros muchos pedaços de Casas yentrellos vna por tada y Cuarto dela

- 28. mja la qual mato avnbachiller Contreras / y delas demas la mayor parte e $\int \tan$

- 29. tan açolbadas de çieno que no se pueden abitar. final men te murie

Y la que consideramos tipográfica se encuentra con punto, diagonal, cruz y guion.

Ej. 8

- 7. /la Casa dexpoual saluatierra all's? yenella no murieron mas de ocho yn

- $\quad$ 8. dios de serujçio y vna donzellica .//.

Es importante insistir en que delimitar de manera estricta una función es cuestionable, pues, como se observa en el último ejemplo, la entonación inicial y la final de la cláusula tiene dos momentos, ascendente y descendente; además, a nivel semántico se indica un 'sentido completo' y a nivel sintáctico una oración compleja; incluso tipográficamente se señala la división del texto.

En suma, los signos que documentamos son: el punto, la diagonal, los dos puntos, el paréntesis, el guion y el espacio en blanco. De los signos y recomendaciones que proporciona López de Velasco para la escritura manuscrita tenemos: el punto, los dos puntos y la diagonal. 


\section{(.), (/), (:), [( )], ( -$),[$ e.b. $]$}

Manuscritos

\section{$(),.(:)$ \\ $(/)$}

López de Velasco

Las señales recomendadas por López de Velasco para la escritura manual se ven claramente rebasadas por los registros; de hecho, para tener la puntuación recomendada para los impresos hacen falta la coma, el punto y coma, el apóstrofo, los admirativos e interrogativos y el iphen.

Como se ha podido observar, el punto, que se emplea para indicar fin de periodo, y la diagonal que se usa en "fin de las cláusulas y razones", amplían sus funciones. Los dos puntos no se emplean como indica López de Velasco en "los miembros más principales, donde se quisiere dar a entender que la razón passa adelante", sino que, como hemos visto, introduce un discurso. El paréntesis aísla una oración explicativa 'que se puede quitar'. Velasco sólo refiere que el espacio en blanco 'va entre palabras', lo cual no se corresponde con lo que se observa en estos escritos. Y el guion no es mencionado por él. ${ }^{15}$

Encontramos, por otra parte, que la diagonal es el signo más empleado con un total de 197 recurrencias, distribuidas entre las distintas funciones: marcando inicio y fin de párrafo, fin de cláusula, ante conjunciones, antecediendo concesivas, circunstanciales, subordinadas y condicionales. El siguiente signo con más recurrencias es el punto con 173 casos.

En los documentos formales se observa más diversidad en los signos empleados y el punto es el signo de puntuación más frecuente; en cambio, en los semiformales predomina la diagonal, y en los informales apenas se emplean el punto y la diagonal (cuadro de registro 1).

\footnotetext{
${ }^{15}$ En cambio, vemos que Antonio de Torquemada reconoce el guion aunque no encontramos su uso. Que para Venegas, los dos puntos, que él llama articulo, se ponen 'en lugar de conjunción', es decir, en coordinadas; que la diagonal puede no ir sencilla sino acompañada con punto (./.), (./), y sirve también en lugar de conjunción. Francisco de Robles reconoce el punto en las enumeraciones. Por último, Nunes de Lião recomienda los dos puntos para referir las palabras de otro.
} 


\begin{tabular}{|l|c|c|c|}
\hline & Formal & Semiformal & Informal \\
\hline Punto & 83 & 82 & 8 \\
\hline Diagonal & 50 & 122 & 25 \\
\hline Guion & 15 & 0 & 0 \\
\hline Paréntesis & 2 & 0 & 0 \\
\hline Espacio bl. & 5 & 0 & 0 \\
\hline Dos puntos & 0 & 1 & 0 \\
\hline
\end{tabular}

Cuadro 1. Registro

La comparación diatópica es interesante, pero hay que puntualizar algunas cuestiones que podrían condicionar el resultado obtenido: primero, la frecuencia de los recursos empleados tiene gran diferencia entre unos y otros manuscritos debido principalmente a su extensión; segundo, los documentos peninsulares pertenecen a mujeres y los americanos a hombres; tercero, ls formación educativa de los escribientes es, al parecer, significativa, y está en relación con la variedad de signos empleados.

Después de estas consideraciones, lo que se encuentra es que el punto y la diagonal son de uso común en ambos lados del Atlántico. El recurso de la combinación de ambos signos lo utilizan Leonor en Sevilla, la reina Juana en Madrid, Nuño en México y el Obispo en Guatemala, y aparece en documentos formales, semiformales e informales. El licenciado Sandoval y el Obispo ofrecen más variedad de signos, pues emplean los dos puntos, los paréntesis, los espacios en blanco y marcan de manera sistemática el inicio y fin de párrafo con raya baja y/o diagonal con punto, aquí se trata de documentación formal y semiformal (Cuadro 2. Diatopía).

Podemos ver que la diagonal atribuida a Torquemada y reconocida por Lopez de Velasco - que la muestra en combinación con punto- es de uso común y frecuente, así como el punto. Se incluyen de manera limitada los dos puntos que este autor recomienda para la escritura de mano; además del espacio en blanco, los paréntesis y otras marcas como la raya baja que se ubicarían dentro de un grupo de signos más amplio y que son 


\begin{tabular}{|c|c|c|c|c|c|}
\hline \multicolumn{3}{|c|}{ Península } & \multicolumn{3}{|c|}{ Nueva España } \\
\hline $\begin{array}{l}\text { Leonor } \\
\text { del Río }\end{array}$ & Sevilla & $\begin{array}{l}(.),(/) \\
\text { Combina } \\
\text { punto y } \\
\text { diagonal (/.), } \\
(. / .)\end{array}$ & $\begin{array}{l}\text { Nuño de } \\
\text { Guzman }\end{array}$ & Méx. & $\begin{array}{l}(.),(/) \\
\text { Combina punto y } \\
\text { diagonal }(. /)(/ .)\end{array}$ \\
\hline $\begin{array}{l}\text { Juana } \\
\text { Vázquez }\end{array}$ & Trujillo & (.) & $\begin{array}{l}\text { Lic. } \\
\text { Sandoval }\end{array}$ & Méx. & $\begin{array}{l}(.),(/), \\
(:)\end{array}$ \\
\hline Reyna & Madrid & $\begin{array}{l}(.),(/) \\
\text { Combina } \\
\text { punto y } \\
\text { diagonal (/.) }\end{array}$ & Obispo 1 & Guat. & $\begin{array}{l}(.),(/), \\
(\mathrm{i} / \mathrm{f}),(\mathrm{e} . \mathrm{b}) \\
\text { Combina } \\
\text { diagonal y punto } \\
(/ .),(. / / .)\end{array}$ \\
\hline & & & Obispo 2 & Guat. & $\begin{array}{l}(.),(/), \\
\left(\mathrm{i} / \mathrm{f} \_\right),(),(\mathrm{e} . \mathrm{b})\end{array}$ \\
\hline
\end{tabular}

Cuadro 2. Diatopía

empleados por los escribientes con mayor formación: el obispo y el licenciado.

\section{Conclusiones}

Somos conscientes de que este trabajo se encuentra limitado, pues apenas presentamos una descripción de lo que se documenta en siete escritos de registro diferente y diatopía dispersa (Nueva España, Capitanía de Guatemala y Península: Trujillo, Sevilla y Madrid). Aunque esta descripción resulte aún insuficiente para arrojar resultados que respondan a la interrogante sobre el uso de la puntuación en la documentación no destinada a la publicación - de registro formal, semiformal e informal y diatopía más global-, y sea necesario confrontar lo aquí descrito no solo con un ortógrafo, sino con el conjunto de gramáticos que hicieron el camino de nuestras gramáticas y ortografías -inclúyase también la puntuación-, es posible señalar, a ma- 
nera de conclusión, dos cosas: a) que hemos encontrado las mismas funciones señaladas por López de Velasco citadas arriba - una semántico-sintáctica, otra enunciativa y prosódica, y otra también semántico-sintáctica; ${ }^{16}$ las mismas que Sebastián Mediavilla diferencia en usos que priorizan la estructura sintáctica, otros que enfatizan la semántica, las que indican aspectos prosódicos, además de aquellas solamente tipográficas.

Desde este punto de partida, pensamos que, por lo menos en estos siete documentos, lo que aparece como prioritario no es el signo en cuanto tal, sino las funciones que en el español se hacen necesarias para la comprensión del contenido, por lo que estas aparecen señaladas con cualquiera de las posibilidades de representación con que se cuenta; aunque, claro, algunos signos adquieren carta de naturaleza y su uso se particulariza en una función determinada.

Concluyo citando a Sebastián Mediavilla, cuyas investigaciones, desde mi punto de vista, son básicas para este tema:

Como se podrá apreciar [...] las normas para la puntuación van precisándose poco a poco y universalizándose, gracias a la uniformidad que trae consigo la imprenta, pero a lo largo de los siglos XVI y xvir se asiste a una notable transformación que no será tanto de las normas cuanto de los sistemas y las formas de los signos [...] Los usos dependerán de la cultura y buen criterio de que disponga aquel que deba asumir la responsabilidad de la puntuación con que el libro salga de la imprenta (2000: 66).

${ }^{16}$ Para comodidad, reescribimos nuestra cita: La puntuación tiene que ver con tres funciones: la primera de tipo semántico-sintáctica: "apartar los miembros de la oración dudosos"; la segunda, enunciativa o prosódica: "señalar los lugares donde el aliento, y voz, hablando, o leyendo, hace pausa"; y la tercera nuevamente de tipo semántico-sintáctica: "distinguir las cláusulas, partes y miembros dellas". 


\section{Bibliografía}

Blanche-Benveniste, Claire (1998), Estudios lingüísticos sobre la relación entre oralidad y escritura, Barcelona, Gedisa. Frenk, MARgit (1983), "La ortografía elocuente (testimonios de lectura oral en el Siglo de Oro)", en AHI, Actas VIII, Centro Virtual Cervantes, cvc.cervantes.es/literatura/aih/pdf/08/ aih_08_1_058.pdf [consultado el 25 de mayo de 2013].

Esteve Serrano, Abraham (1982), Estudios de teoría ortográfica del español, Murcia, Universidad de Murcia.

Jiménez Patón, Bartolomé ([1614] 1965), Epítome de la ortografía latina, y castellana e Instituciones de la gramática española, edición de Antonio Quilis y Juan Manual Rozas. Madrid, Consejo Superior de Investigadores Científicos.

Koch, Peter y Wulf Oesterreicher (2007), Lengua hablada en la Romania: español, francés, italiano, Madrid, Gredos (Biblioteca Románica).

López de Velasco, JuAn (1582), Orthographia y pronunciación castellana, Burgos.

Luna Traill, Elizabeth, Alejandra Vigueras Ávila y Gloria Estela Báez Pinal, (2005), Diccionario básico de lingüística, México, Universidad Nacional Autónoma de México.

Madariaga, Pedro (1565), Arte de escribir ortografía de la pluma, y honra de los profesores de este magisterio, 2a impresión 1767, Madrid, D. Antonio de Sancha.

MARTínez Marín, JUAN (1992), "La ortografía española: perspectiva historiográfica”, Cauce, 14-15, pp. 125-134.

Peñalver Castillo, Manuel (2015), "El Prontuario de Ortografía de la lengua castellana (1844). Antecedentes y consecuentes", Anuario de Letras. Lingüística y Filología, III, 2, pp. 313-356.

Real Academia Española (2011), Ortografía de la lengua española, México, Planeta Mexicana.

Real Academia Española (2013), Diccionario de la lengua española, 22a ed. [consultado en abril-mayo de 2013, www. rae.es]. 
Rubio Flores, Antonio R. (2006), “Una teoría de la puntuación en la "Carta-Proemio" de la Eneida de Don Enrique de Villena: el valor del resuello", Revista de Poética Medieval, 17, pp. 195-214.

SAntiago, Ramón (1998), "Apuntes para la historia de la puntuación en los siglos XVI y XVII", en José Manuel Blecua, Juan Gutiérrez y Lidia Sala (eds.), Estudios de grafemática en el dominio hispano, Salamanca, Instituto Caro y CuervoUniversidad de Salamanca, pp. 243-280.

Sebastián Mediavilla, Fidel (2007), "La puntuación del Quijote”, Anales Cervantinos, 39, pp. 101-145.

Sebastián Mediavilla, Fidel (2008), "Mateo Aleman y la puntuación del Guzmán de Alfarache", Revista Lectura y Signo: Revista de Literatura, 3, 1, pp. 237-270.

Sebastián Mediavilla, Fidel (2007), Puntuación, humanismo e imprenta en el Siglo de Oro, Vigo, Pontevedra, Academia del Hispanismo.

Sebastián Mediavilla, Fidel (2000), La puntuación en los Siglos de Oro: Teoría y práctica, Tesis doctoral, Barcelona, Universidad de Barcelona.

Venegas, Alejo (1531), Tractado de orthographia y accentos en las tres tres lenguas principales, Toledo, Impreso por Lázaro Salvago Ginoués. 